\title{
Oral vibrotactile stimulation: A method for monitoring change in lingual sensitivity as a function of time
}

\author{
DONALD J. FUCCI, ANN P. CURTIS, and MARTHA M. HARNACK \\ School of Hearing and Speech Sciences, Ohio University, Athens, Ohio 45701
}

\begin{abstract}
This study describes assessment procedures used to monitor the change in lingual sensitivity of a 40-year-old partial glossectomized female subject over a period of time. Oral vibrotactile thresholds were obtained from the right, midline, and left sections of the anterior dorsum of the tongue once a week for 7 weeks while the subject was receiving speech therapy. Improvement in sensitivity was noted for all three lingual sections. The implications for using oral vibrotactile threshold measurements for monitoring purposes were discussed.
\end{abstract}

Partial glossectomy has been used successfully as a surgical adjunct in selected patients with macroglossia, apertognathia, tongue thrust, lisping, and combinations of these (Allison, Miller, Troiana, \& Wallace, 1971). The degree of articulatory impairment following glossectomy depends on the amount of tissue removed and its location (Messengill, Maxwall, \& Pickrell, 1970). Rehabilitation of partial or total glossectomized patients through systematic speech training procedures and appropriate practice has produced satisfactory results in speech, chewing, and swallowing, respectively (Brodnitz, 1965). Therapy for cases having had glossectomy has included mainly stretching, massage, and resistive exercises to increase the biologic functions of chewing, sucking, and swallowing (Messengill et al, 1970). Improvement of these functions results in definite changes in speech articulation and intelligibility (Greene, 1937).

Subject for this study was a 40-year-old partial glossectomized female. Upon initial examination by a dental surgeon, the subject presented mandibular prognathism and hypertrophy of the tongue, which led to impairment of speech intelligibility. Subject received surgery in August, 1973, at which time an intraoral sagittal split osteotomy was performed for correction of the prognathism. At the same time, a partial glossectomy was done for reduction of tongue size. In October of that year, fixation appliances were removed and the subject was then referred to a periodontist for equilibration of the postoperative occlusion and treatment of chronic periodontal disease. Subject was referred to the Ohio University Speech and Hearing Clinic for subsequent therapy for the speech intelligibility.

*This paper is sponsored by James L. Bruning, who takes full editorial responsibility for it.

\section{METHOD}

Subject was seen for speech therapy starting in January and continued attending two 1 -h $\mathrm{h}$ weekly sessions for a total of 7 weeks. A portion of the second hour of each weekly session was used to obtain vibrotactile thresholds from the right, midline, and left sections of the anterior dorsum of the tongue. These thresholds were obtained to monitor changes in lingual tactile sensitivity that might be occurring during the 7-week period. Equipment and procedures employed for testing are described in detail by Fucci and Kelly (1972). Weekly thresholds were obtained at the frequencies of $125,250,300$, and $500 \mathrm{~Hz}$. These frequencies have been shown to be ones to which the oral tactile sense is most responsive (Fucci, 1972). An ascending-pulsed method of stimulation was employed to obtain the thresholds (Hall, Fucci, \& Arnst, 1972). Thresholds were obtained for each frequency and then averaged across frequencies so that one threshold figure would exist for each of the three lingual sections tested.

\section{RESULTS AND DISCUSSION}

The results of the monitoring procedure are shown in Figure 1. It can be seen that a definite change took place in lingual sensitivity during the 7 -week period. The first week thresholds for the right, midline, and left lingual sections respectively were $6.1,3.7$, and 3.3 microns. The seventh week thresholds for the right, midline, and left lingual sections, respectively, were $1.2,1.0$, and .9 microns. These changes represent an increase in sensitivity of 4.9 microns for the right lingual section, 2.7 microns for the midline lingual section, and 2.4 microns for the left lingual section.

It is of interest to note that the right lingual section demonstrated poorer thresholds throughout the testing period than did the midline section, which in turn demonstrated poorer thresholds than the left lingual section. These findings were in agreement with the subject's subjective appraisal of lingual sensitivity with respect to the ability to correctly place the tongue against other portions of the oral cavity upon direction from the therapist. 


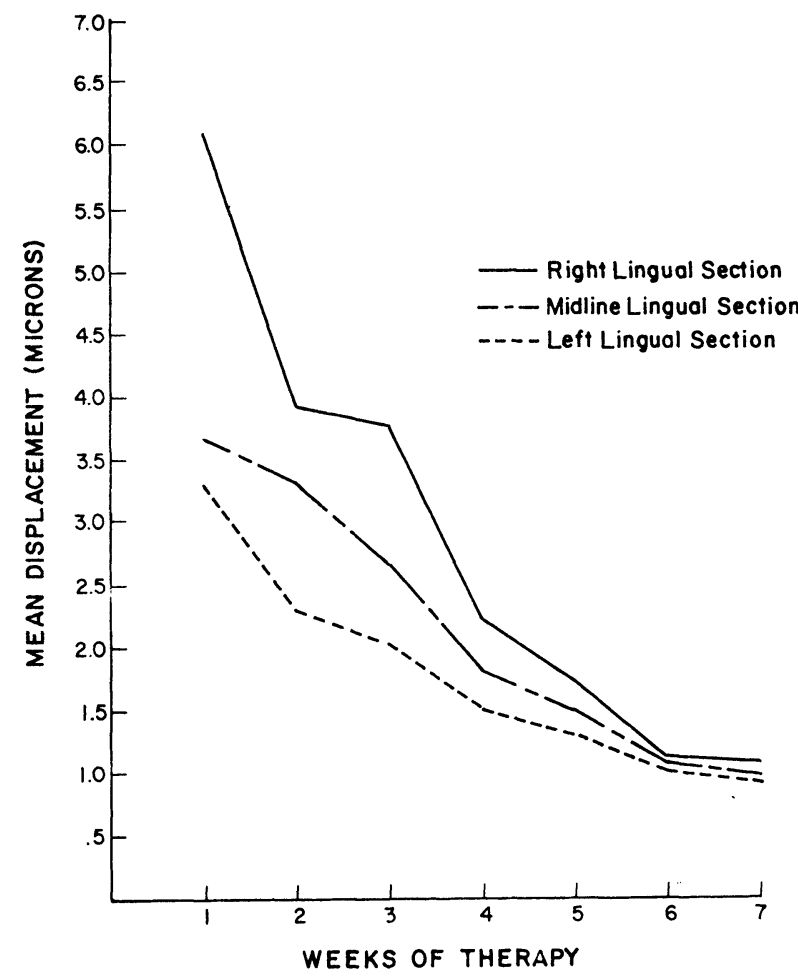

Figure 1. Summary of threshold data (microns) characteristic of averaged findings across frequencies during the 7-week therapy period.

The sixth and seventh week threshold results were in relatively close agreement with normative data obtained on 110 normal speaking adults (Telage, Fucci, \& Arnst, 1972). Subject was demonstrating normal intelligibility by the sixth and seventh weeks and was dismissed.

The reasons for the improved lingual sensitivity could have been many and varied. They could have been due to the speech therapy, the actual threshold testing, which also provided lingual vibrotactile stimulation, natural sensory improvement as a result of time, or some combination of these factors. There were possibly even other factors not considered by all who were involved. The vibrotactile threshold assessment did provide a way to monitor the change in lingual sensitivity over time, however, and in this case it was showing a progression toward established normative information. Once the interpretation and meaning of this material becomes an integral part of a speech therapist's plans, it could eventually become as important as the audiogram presently is to the audiologist dealing with a client who has had a stapedectomy or some other form of ear surgery.

\section{REFERENCES}

Allison, M. L., Miller, C. W., Troiana, M. F., \& Wallace, W. R. Partial glossectomy for macroglossia. Journal of the American Dental Association, 1971, 82, 852-857.

Brodnitz, F. S. Vocal rehabilitation. Rochester: Custom Printing, 1965.

Fucci D. J. Oral vibrotactile sensation: An evaluation of normal and defective speakers. Journal of Speech and Hearing Research, 1972, 15, 179-184.

Fucci, D. J., \& Kelly, D. H. New instrumentation for research on vibrotactile sensitivity of the tongue. Review of Scientific Instruments, 1972, 43, 1748-1751.

Greene, J. S. Speech defects and related oral anomalies. Journal of the American Dental Association, 1937, 24m 1969-1974.

Hall, D., Fucci, D., \& Arnst, D. Vibrotactile stimulation: An investigation of psychophysical methods for establishing threshold. Perceptual and Motor Skills, 1972, 34, 891-898.

Messengill, R., Maxwell, S., \& Pickrell, K. An analysis of articulation following partial and total glossectomy. Journal of Speech and Hearing Disorders, 1970, 35, 170-173.

Telage, K., Fucci, D., \& Arnst, D. Normative study of oral vibrotactile sensitivity. Perceptual and Motor Skills, 1972, 35 , 671-676.

(Received for publication September 17, 1974.) 